\title{
Rudimentary Horn Pregnancy: A Rare First Trimester Acute Presentation
}

\author{
Divya Pandey ${ }^{*}$, Brajendra N. Tripathi $^{2}$, Pallav Mishra ${ }^{3}$
}

\begin{abstract}
Introduction: Rudimentary horn pregnancy (RHP), an entity with a diagnostic dilemma is known to rupture most commonly in second trimester, sometimes in early third trimester. This case of ectopic rudimentary horn (RH) presented acutely in the first trimester.

Case Presentation: A 30 years old $\mathrm{G}_{3} \mathrm{P}_{2} \mathrm{~L}_{2}$ with previous two vaginal deliveries presented with acute abdominal pain and features of hemorrhagic shock at 8 weeks 5 days gestational age. With clinical diagnosis of ruptured ectopic pregnancy, the patient immediately underwent laparotomy, which revealed hemoperitoneum with a unicornuate uterus along with ruptured RHP. RH excision with ipsilateral salpingectomy and right tubal ligation was done and the maternal life salvaged.

Conclusion: High index of suspicion based on clinical history and examination complemented with diagnostic radiological modalities can help in prenatal and pre-rupture diagnosis of RHP, hence its prompt treatment to alleviate scope of any emergency situation and the disastrous consequences leading to maternal morbidity and mortality.

Keywords: Ectopic pregnancy, First trimester, Rudimentary horn pregnancy, Rupture
\end{abstract}

\section{Introduction}

The rudimentary horn pregnancy (RHP) (incidence of $1 / 10,000$ to $1 / 40,000$ ) usually ends up in rupture of gravid horn in second or third trimester, leading to potentially fatal hemorrhage. Modern modalities can diagnose it in a very early pre-rupture stage, thus making medical management possible (1). Acute presentation at an early gestation is a very rare phenomenon. This case reports such an early presentation in the first trimester at 8 weeks, which has not been reported so far in literature.

\section{Case Presentation}

A 30-year-old third gravida with previous two institutional vaginal deliveries, last delivery 3 years back, presented with acute abdominal pain with hemorrhagic shock following 2 months amenorrhea. Her urine pregnancy test was positive, gestational age being 9 weeks by last menstrual period and 8 weeks 5 days by a scan done at 7 weeks reporting an intrauterine pregnancy. With normal menstrual history and history of barrier contraceptive usage, she had first antenatal visit about 2 weeks back to an obstetrician, who without pelvic examination advised a dating scan. She was pale, had cold extremities, blood pressure of $88 / 50 \mathrm{~mm} \mathrm{Hg}$ and pulse rate of $120 /$ minute. There was marked lower abdominal guarding and tenderness with dull percussion note in both flanks. Vaginal examination revealed a soft, bulky uterus deviated to the right side with a tender, soft to firm mass through left fornix. Her hemoglobin level was $6 \mathrm{gm} / \mathrm{dl}$. Immediate trans-vaginal sonography done in casualty room showed a normal size uterus with a heterogeneous complex cystic mass of $5.5 \times 6 \mathrm{~cm}$ in left adnexa and evidence of free fluid in peritoneal cavity. With provisional diagnosis of ruptured ectopic pregnancy with shock, the patient immediately underwent emergency exploratory laparotomy along with simultaneous volume resuscitation. There was about 1 litre hemoperitoneum. The uterus was unicornuate with absolutely normal right adnexa. A non-communicating rudimentary horn (RH) on left side carrying the ectopic sac was in the process of rupture with surrounding omentum adhered to it, with area of active bleeding (Figure 1). The horn was connected to the left wall of the uterus by a thick fibrous band with the ipsilateral adnexa stretched over it. The RH with left tube and contents of ectopic pregnancy was excised along with right tubal ligation (Figure 2). She received 3 units of blood transfusion and was discharged on seventh post-operative day. Follow up at 1 month was normal. Histopathology report further confirmed the diagnosis.

\section{Discussion}

In non-pregnant state, $\mathrm{RH}$ is the most difficult congenital mullerian malformation to be detected in a silent state. 


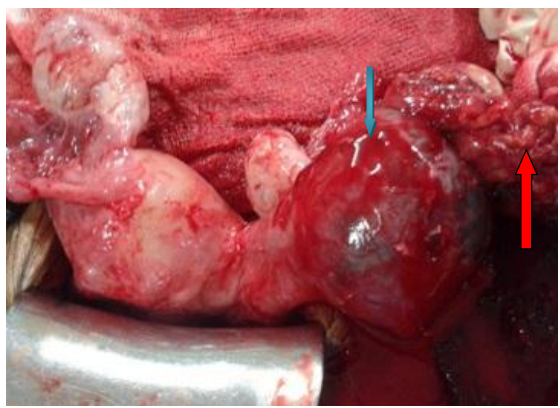

Figure 1. Per-operative picture showing the rudimentary horn on left side with ectopic pregnancy (blue arrow) in the process of rupture with active bleeding; bleeding site being partially sealed by surrounding omentum (red arrow).

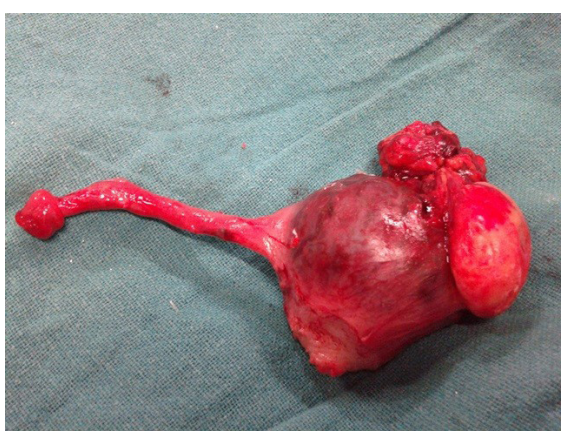

Figure 2. Post-operative picture of the specimen of resected rudimentary horn along with the ipsilateral tube.

Unicornuate uterus with a $\mathrm{RH}$ often presents with recurrent first trimester abortions (5\%-10\%), a second trimester loss (25\%), or is incidentally discovered during an infertility work-up. There may be wide spectrum of clinical presentation ranging from a trivial dysmenorrhea in adolescence to intractable vague pelvic pain in a parous woman (2). Hematometra is uncommon due to lack of menstrual shedding from associated aplastic or hypoplastic endometrium. However, our patient had normal gynecological history with previous normal reproductive outcomes.

RHP is due to the intraperitoneal transmigration of the sperm or contra-lateral tubal pick up of the fertilized ovum in the peritoneal cavity. The musculature of $\mathrm{RH}$ is thin and mal-developed and endometrium is non-functional invoking a pathological placentation. Natural history of RHP involves rupture occurring in $80 \%-90 \%$ in second or early third trimester; however term is reached by only $10 \%$ with a fetal salvage rate of $2 \%$ (3-5). Abdominal pregnancies reaching term after rupture of $\mathrm{RH}$, with its placenta deriving fetal blood supply from RH myometrium, have been described in the literature where the pregnancy outcome was a live fetus by cesarean section (6-8). There has been a report of a successful birth of twins by cesarean section, where one of the fetuses was in the non-communicating $\mathrm{RH}(9)$.

What is unusual in our case is that, despite the functional endometrium in the $\mathrm{RH}$, the patient had no symptoms of dysmenorrhea, hematometra, or pelvic endometriosis. This could be explained by extremely limited endometrial shedding because of a severely hypoplastic uterine cavity.
Secondly a very early rupture due to the thin horn wall and a hypoplastic cavity was evident. Even the only scan which was done, failed to detect the ongoing RHP. Thorough search of electronic database of Pubmed and Google Scholar from 1950 to 2014 using MeSH terms "rudimentary horn pregnancy", "acute", "rupture", and "first trimester" did not show any case of such early first trimester acute presentation (8 weeks).

Although having a diagnostic dilemma till rupture, high clinical index of suspicion supported by radiological diagnostic modalities may help to clinch and treat it at pre-gestational or pre-rupture state before it causes emergency leading to maternal morbidity or mortality. However, in case of emergency prompt diagnosis and immediate removal of the RH is lifesaving.

Even a simple pelvic finding of a soft, lateral mass corresponding to a small gravid horn with a normal uterus should raise the clinician's doubt. The obstetrician where the patient had her first antenatal visit could have caught the condition by doing a simple pelvic examination. But this is subjective needing clinical expertise. While pre-pregnancy diagnosis can be reached by hysterosalpingography, laparoscopy or hysteroscopy, antenatal diagnosis can be made by a simple trans-vaginal ultrasonography. Expertise is needed to detect it by ultrasound at pre-rupture state. Even in our case the diagnosis was missed at 7-week scan.

Ultrasound can confirm RHP by revealing pregnancy circumscribed by a variable thickness of myometrium. This myometrial thickness is not seen in tubal ectopic pregnancy (10). Three dimensional ultrasound can be of special help in detecting mullerian anomalies. However, the diagnosis can be elaborated by using MRI, but could not be done in our case owing to the emergency state. Radio-imaging modalities thus help in timely management.

The standard treatment of RHP, normal or complicated, involves fetal extraction with or without excision of $\mathrm{RH}$ along with the ipsilateral tube. Combined medical and surgical treatment has also been suggested (1). Certain other authors' even proposed prophylactic resection on diagnosis or as incidental finding to avoid any future obstetric insult, but future pregnancy will then require close follow up (2).

\section{Conclusion}

The clinicians should bear high index of suspicion for this entity even in patients with normal obstetric and gynecological history for timely diagnosis using the existing radiological modalities for prompt treatment to avoid any morbidity or mortality.

\section{Ethical issues}

Written informed consent was obtained from the patient for publication of this case report and accompanying images.

\section{Financial support}

No financial support was needed for this case reporting. 


\section{Conflict of interests}

The authors declare no conflict of interests regarding this manuscript.

\section{Acknowledgments}

We would like to thank all our colleagues who helped us in this study.

\section{References}

1. Park JK, Dominguez CE .Combined medical and surgical management of rudimentary uterine horn pregnancy. JSLS 2007;11(1):119-22.

2. Malik R, Radhika AG, Singh A, Radhakrishnan G, Aggarwal R. The perplexing entity of rudimentary uterine horn .Open J Obstet Gynecol 2011;1:217-20. doi:10.4236/ojog.2011.14042.

3. Basbug M, Soyuer I, Aygen E. Placenta accreta associated with rupture of a rudimentary horn pregnancy. Int J Gynaecol Obstet 1997;57(2):199-201.

4. Kuscu NK, Lacin S, Kartal O, Koyuncu F. Rupture of rudimentary horn pregnancy at the 15th week of gestation: a case report. Eur J Obstet Gynecol Reprod Biol 2002;102(2):209-10.

5. Rolen AC, Choquette AJ, Semmens JP. Rudimentary uterine horn: Obstetric and gynecologic complications. Obstet Gynecol 1966;27:806-13.

6. Kawthalkar AS, Gawande MS, Jain SH, Joshi SA, Ghike SD, Bhalerao AV. Rare case of live birth in a ruptured rudimentary horn pregnancy. J Obstet Gynaecol Res 2011;37(8):1169-72. doi: 10.1111/j.1447-0756.2010.01497.x.

7. Amritha B, Sumangali T, Priya B, Deepak S, Sharadha $\mathrm{R}$. A rare case of term viable abdominal pregnancy following rupture of a rudimentary horn:a case report. J Med Case Rep 2009; 3:38. doi:10.1186/17521947-3-38.

8. Shin JW, Kim HJ. Case of live birth in a noncommunicating rudimentary horn pregnancy. J Obstet Gynaecol Res 2005;31(4):329-31.

9. Nanda S, Dahiya K, Sharma N, Aggarwal D, Singhal SR, Sangwan N. Successful twin pregnancy in a unicornuate uterus with one fetus in the noncommunicating rudimentary horn. Arch Gynecol Obstet 2009;280(6):993-5. doi:10.1007/s00404-0091028-x.

10. Kriplani A, Relan S, Mittal S, Buckshee K. Pre-rupture diagnosis and management of rudimentary horn pregnancy in the first trimester. Eur J Obstet Gynecol Reprod Biol 1995;58(2):203-5.

Copyright $\odot 2015$ The Author(s); This is an open-access article distributed under the terms of the Creative Commons Attribution License (http://creativecommons.org/licenses/by/4.0), which permits unrestricted use, distribution, and reproduction in any medium, provided the original work is properly cited. 